\section{Regenerative Endodontic Treatment as a Retreatment Option for a Tooth with Open Apex - A Case Report}

Maria-Elpida A. Miltiadous, Spyros G. Floratos
Private Practice, Endodontics, Athens, Greece

Correspondence: Spyros G. Floratos, DMD, 13 Koniari Str, Athens, 114 71, Greece, Tel: +30 2106427232 . e-mail: sflo_@hotmail.com
Treatment of non-vital immature teeth with apical periodontitis has always been a challenge in Endodontics. Regenerative endodontic treatment (RET) has been successfully used for the management of these cases. The aim of this study is to present a case of RET used for the retreatment of a previously endodontically treated permanent tooth with an open apex. A 14-year-old boy with a poor endodontic treatment done on his maxillary right central incisor developed symptomatic apical periodontitis. Radiographically, incomplete root development with thin dentinal walls and an open apex were evident. After accessing and removing previous filling materials, the canal was copiously irrigated with $2.5 \%$ sodium hypochlorite. A triple antibiotic paste was placed inside the canal and left for 15 days. After removal of the antibiotic mixture, bleeding was induced into the canal by passing a hand file out of the apex. A collagen membrane barrier was placed over the blood clot, followed by sealing with mineral trioxide aggregate. Access was sealed with permanent filling materials. Clinical examination at 12, 24 and 36 months revealed no symptoms. Radiographic examination showed resolution of the periapical lesion and apical closure. Sensitivity tests with cold and an electric pulp test elicited a negative response at all recall periods. On the basis of long-term results, RET may be an effective option for the retreatment of an immature permanent tooth with a failed previous treatment and periapical periodontitis.
Key words: endodontic retreatment, immature permanent tooth, regenerative endodontic treatment.

\section{Introduction}

Endodontic treatment of necrotic immature teeth has always been significantly challenging due to the disrupted development of root canal walls and open apices. The traditional treatment of choice in these cases includes a multiple-visit, long-term application of calcium hydroxide to promote the formation of an apical calcified barrier $(1,2)$. Another treatment of necrotic teeth with open apices is the placement of an artificial apical barrier using mineral trioxide aggregate (MTA). These techniques have been proven to be highly successful however both apexification and artificial apical barriers prevent the continuation of root development and thickening of dentinal walls, thus making teeth susceptible to root fractures $(3,4)$.

Regenerative endodontic treatment (RET) is a biologically based alternative method of treating necrotic immature teeth and allowing for continuation of root development (5). The term "revascularization" was introduced to describe a treatment protocol for necrotic immature permanent teeth with apical periodontitis. Revascularization consists of three basic steps. Primarily, the root canal is disinfected without mechanical instrumentation with the use of copious sodium hypochlorite $(\mathrm{NaOCl})$ irrigation followed by the filling of the canal with a mixture of ciprofloxacin, metronidazole and minocycline (6). After successful disinfection during which clinical signs and symptoms of infection subside, the antibiotic paste is removed and a blood clot is produced. Finally, MTA is used to seal the root canal to the level of the cementoenamel junction and a coronal restoration is placed. This technique has demonstrated its therapeutic value by promoting varying degrees of root elongation, thickening of root canal walls, apical closure and periradicular healing $(7,8)$.

The survival rate of immature teeth treated with RET $(100 \%)$ and MTA apexification (95\%) were significantly greater than the survival rates of the calcium hydroxidetreated teeth (77.2 \%) (9). Furthermore, with regard to the increase of root length and width, the percentages observed in the RET group (14.9\% and $28.2 \%)$ were significantly greater than those reported in the MTA apexification group (6.1\% and $0.0 \%$ ) and calcium hydroxide apexification group $(0.4 \%$ and $1.5 \%)$ (9). Based on these encouraging results, RET consists a successful treatment option for non-vital immature teeth.

There are several studies and numerous case reports supporting the use of RET as the treatment of choice in cases of necrotic immature teeth with periapical periodontitis $(6,7)$. Nevertheless, a case of using RET as a retreatment option has not been reported up to the present time. The aim of this study is to present a case report with the use 
of RET as a retreatment option of an immature tooth with previous failed endodontic treatment and symptomatic apical periodontitis.

\section{Case Report}

A 14-year-old Caucasian male was referred to the private office by an oral surgeon for evaluation and treatment of tooth \# 11 (maxillary right central incisor). He complained of an intermittent pain upon biting on the tooth. Patient's medical history revealed no significant medical pathosis. The dental history revealed trauma on tooth \# 11 five years before, which resulted, according to his mother's statement in a crown fracture with pulp exposure. The tooth was immediately treated by his general practitioner with an endodontic treatment and a composite resin restoration. Clinical examination revealed moderate palpation pain, severe percussion pain and intraoral swelling at the buccal alveolar mucosa of the right maxillary central incisor. Crown discoloration of tooth \#11 was also apparent (Fig. 1A). Periodontal probings were within normal limits and there was no mobility of the tooth. Radiographic examination revealed a previous endodontic treatment, open apex and thin dentinal walls and an apparent radiolucency around the apex of tooth \#11. Noticeably, a radiopaque mass in the vicinity of the root apex could be observed in the preoperative radiograph (Fig. 1B). Based on the history, clinical tests and radiographs, a diagnosis of previous root canal treatment of an immature permanent tooth with symptomatic periapical periodontitis was established. Regenerative endodontic treatment (RET) was determined as a retreatment option for tooth \#11. Written consent was obtained for the treatment plan which was signed by the patient's parent.

After local anesthesia (Ubistesin 4\%, 3M ESPE, Germany) and rubber dam isolation, the tooth was accessed and gutta-percha points were removed by using an $80 \mathrm{~K}$ file (Dentsply Maillefer, Ballaigues, Switzerland). Working length was determined radiographically (Fig. 1C). The root canal was gently irrigated with $24 \mathrm{ml}$ of $2.5 \% \mathrm{NaOCl}$, without any instrumentation. $\mathrm{NaOCl}$ was delivered via a 30-gauge closed end, side vent needle (Canal Clean, Biodent Co., Ltd., Paju-city Guynggi-do, Korea), positioned $1 \mathrm{~mm}$ short of working length. $\mathrm{NaOCl}$ was activated for $60 \mathrm{~s}$ by use of ultrasonics (25K, CK tip, BCtL Biotech, Philadelphia, PA). After the canal was dried with paper points, a triple antibiotic paste consisting of $500 \mathrm{mg}$ ciprofloxacin powder, $500 \mathrm{mg}$ metronidazole powder and $500 \mathrm{mg}$ amoxicillin powder was introduced in the canal with a lentulo spiral and filled the canal up to the level of the cementoenamel junction (CEJ). The access cavity was sealed with $4 \mathrm{~mm}$ of Cavit (ESPE, Seefeld, Germany).

The patient returned 15 days later, reporting that clinical symptoms had subsided. Clinical examination revealed no palpation or percussion pain and there was no intraoral swelling. After local anesthesia with 3\% mepivacaine with no vasoconstrictor, the tooth was isolated using a rubber dam and accessed. The tri-antibiotic paste was removed with irrigation with $12 \mathrm{ml}$ of $2.5 \% \mathrm{NaOCl}$ followed by $12 \mathrm{ml}$ of 17\% EDTA solution and dried with sterile paper points. After the root canal appeared to be clean and dry, a sterile $20 \mathrm{~K}$-File was passed $2 \mathrm{~mm}$ out of the apex and gently irritated the periapical tissues to induce blood flow in the canal. The intracanal bleeding was controlled at a level of $3 \mathrm{~mm}$ below the CEJ by placement of a sterile cotton pellet for 3 minutes (Fig. 1D). Following that, a sterile collagen membrane barrier (Collacote, Zimmer Dental, Carlsbad, CA USA) was placed on the blood clot by use of new sterile pliers. Mineral trioxide aggregate (MTA, Dentsply, Tulsa, OK, USA) was mixed and placed on the collagen membrane forming a $3 \mathrm{~mm}$ layer (Fig.1E). A moist cotton pellet was placed on top of the MTA and glass ionomer cement was used to seal the access. Two days later the tooth was re-accessed, MTA set was confirmed and the access was sealed with composite resin. A postoperative radiograph was taken (Fig. 1F).

The patient was recalled yearly for clinical and radiographic follow-ups. At two-year follow-up a complete healing of the radiolucent lesion was evident (Fig. 1G). At three-year follow-up session the tooth was asymptomatic and functional (Fig. 1H). The patient reported no recurrence of swelling. The response to the cold test was negative in all follow-up sessions. In radiographic examination at three years apical closure was visible. However, there was no increase in the length and thickness of the canal walls. Cone-beam computed tomography (CBCT) was taken to evaluate the accurate position of the calcification present at the periapical area. The result indicated the radiopaque image was attached with dentin wall in buccolingual direction like a bridge (Fig.11,1J).

\section{Discussion}

RET is a biologically based alternative approach to treat necrotic immature teeth that, unlike apexification and artificial apical barrier techniques, allows continuation of root development (10). The endodontic treatment of permanent teeth with open apex has been a clinical challenge as (11): 1. Mechanical instrumentation of the root canal is difficult because of the anatomy of the immature tooth; 2. An apical seal of the root canal by using traditional filling methods is difficult because of the absence of an apical stop; 3 . Thin root canal walls of immature teeth are susceptible to fracture (4).

For these reasons, disinfection of the canal system relies solely on irrigants and intracanal medications. In 

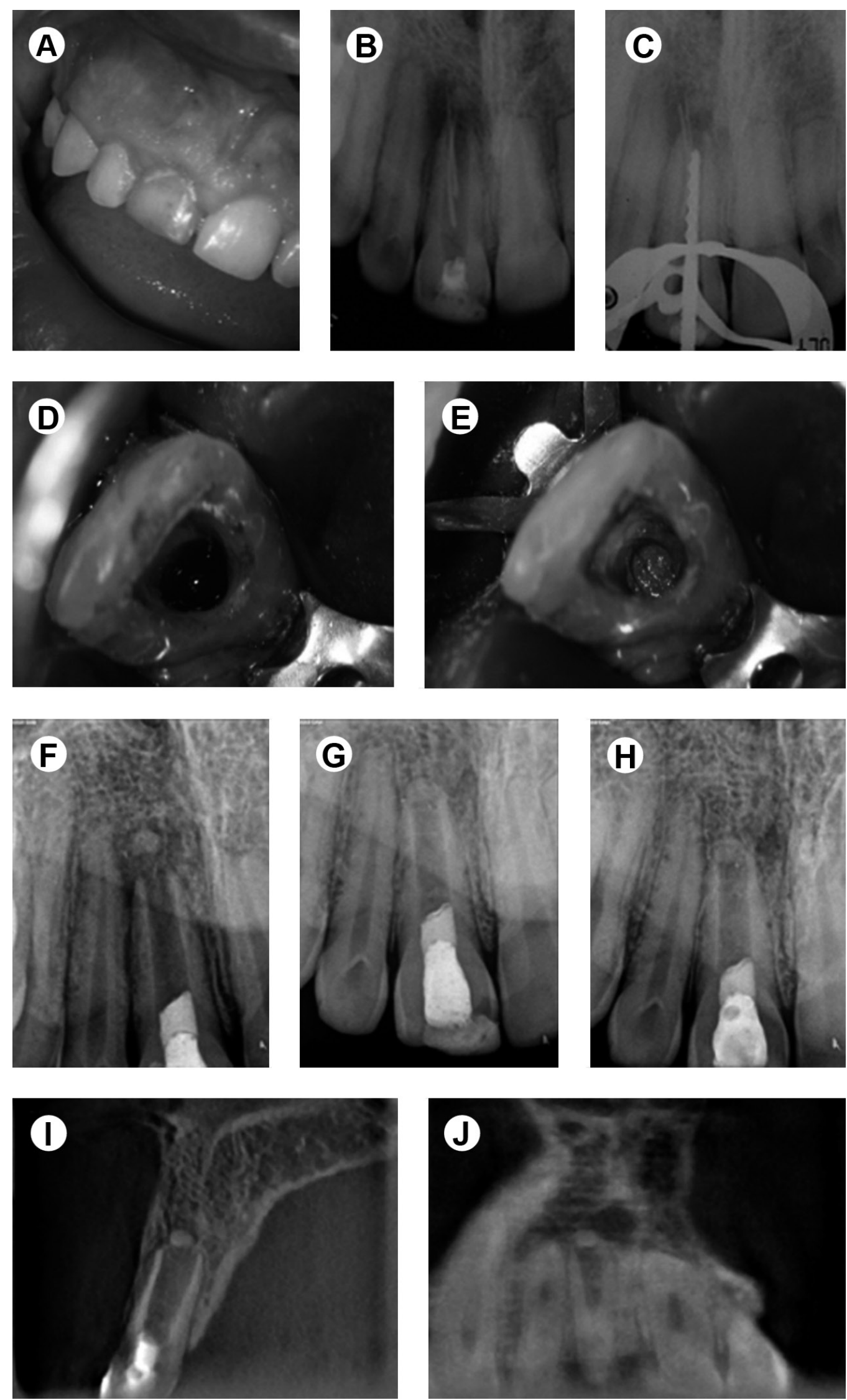

Figure 1. Treatment sequence and recall evaluation. A: preoperative clinical image of the maxillary right central incisor. B: Preoperative radiograph. C: Removal of gutta-percha points and radiographic estimation of working length. D: Canal filled with blood (original magnification, 12x). E: Placement of MTA on the collagen membrane (original magnification 12x). F: Postoperative radiograph. G: Radiograph taken 24 months after treatment. H: Radiograph taken 36 months after treatment. 1: Sagittal view of the CBCT taken at 36 months. J: Coronal view of the CBCT at 36 months 
our case report, $2.5 \% \mathrm{NaOCl}$ and 17\% EDTA were used as chemical irrigants at the second appointment. EDTA has been suggested to be a single irrigant for pulp regeneration in immature necrotic teeth at the second appointment because EDTA was shown to promote stem cells of apical papilla (SCAP) survival (89\% viability) (12). Lower cell viability (74\%) was observed when both sodium hypochlorite and EDTA were used (12). A combination of sodium hypochlorite and EDTA was used in this case, although a significant decrease in viability of stem cells was expected.

SCAP are considered to be a main cell source for pulp regeneration in immature teeth (13). SCAP are defined as mesenchymal stem cell populations residing at the apical papilla around the root apices of immature permanent teeth $(13,14)$. Periodontal ligament stem cells $(15,16)$, bone marrow mesenchymal stem cells $(16,17)$, and some surviving dental pulp stem cells around the root apex may participate in pulp regeneration. It is necessary that all these cells be stimulated to migrate into root canal space. Bleeding induced by passing files beyond the apex was thought to stimulate the migration of adult stem/ progenitor cells into the root canal (18). In the case report presented, no thickening of the dentinal walls occurred and further root development was not evident. This can probably be explained by the fact that copious irrigation with a high concentration of sodium hypochlorite close to the apex done at the previous endodontic treatment may have damaged the SCAP, other progenitor/stem cells or apical vital tissue (19). Another possibility is the fact that persistent canal wall debridement during the primary treatment did not allow for hard tissue deposition and further thickening of dentinal walls. When bleeding was induced in immature teeth, there was up to 600 -fold increase in stem cell markers in canal blood compared with the level in systemic blood (18). Probably the blood that filled the canal space in the case reported here might have been poor in stem cells as SCAP were damaged by the cytotoxic effect of the sodium hypochlorite used during the primary treatment.

In the case presented, although no increase in root length and no increase in root wall thickness was observed, these aspects may not always be achievable, nor are they essential for treatment success after RET is implemented (20). It has to be noted that at the 36-month recall examination, patient did not present any clinical signal or symptom after treatment completion and follow-up that would indicate regenerative endodontic retreatment fail. Therefore, conclusions related to regenerative endodontic retreatment should be drawn based not only on X-ray and computed tomography images, but on clinical signals and symptoms as well (21).
Five types of response of immature permanent teeth with infected necrotic pulp tissue and apical periodontitis were observed when revascularization procedures were implemented: type 1, increased thickening of canal walls and continued root maturation; type 2, no significant continuation of root development and the root apex became blunt and closed; type 3, continued root development and the apical foramen remained open; type 4, severe canal calcification; type 5, hard tissue barrier between the coronal MTA plug and the root apex (22). Following this classification, the case presented was a type 2 tissue response as root formation did not continue and the apex was closed.

The presence of a radiopaque mass in the vicinity of the root apex could be observed in the preoperative radiograph. Similar radiopaque entities were found by other investigators either at the periapical tissues or close to the apex $(7,8)$, or in the canal space shortly after RET was initiated (11). This entity was speculated to be either bonelike tissue and/or cementum-like tissue (11). This calcific tissue in our case gradually approached the apex as healing of the periapical pathology was progressing. Probably this mineral deposition was formed as a reactionary response to the irritation of vital progenitor cells of the apical papilla during the previous endodontic treatment. The three-year periapical radiography showed this tissue was in continuation with the dentinal walls forming an apical barrier whereas the coronal view of the CBCT indicated the radiopaque image was right at the apical point attached with the dentin walls.

RET has been proven to be a better choice for the treatment of a nonvital immature permanent tooth, as opposed to apexification, because of the thickening of dentin wall and continuation of root development. Based on these advantages we selected this procedure as a retreatment option for the case reported. However, the 36-month follow up of the case showed that RET as a retreatment option for immature teeth with a persistent periapical lesion has no clearly favorable results over apexification. Further clinical studies are needed to support this finding.

This case shows that RET could be used as a retreatment option in the case of a failed endodontic treatment on an immature permanent tooth with apical periodontitis.

\section{References}

1. Yousef Saad A. Calcium hydroxide and apexogenesis. Oral Surg Oral Med Oral Pathol 1988;66:499-501.

2. Shabahang $S$, Torabinejad $M$, Boyne PP, Abedi $H$, McMillan P. A comparative study of root-end induction using osteogenic protein-1, calcium hydroxide, and mineral trioxide aggregate in dogs. J Endod 1999;25:1-5.

3. Mente J, Hage N, Pfefferle T, Koch MJ, Dreyhaupt J, Staehle HJ et al. Mineral trioxide aggregate apical plugs in teeth with open apical 
foramina: a retrospective analysis of treatment outcome. J Endod 2009;35:1354-1358.

4. Tuna EB, Dincol ME, Gencay $K$, Aktoren 0 . Fracture resistance of immature teeth filled with BioAggregate, mineral trioxide aggregate and calcium hydroxide. Dent Traumatol 2011;27:174-178.

5. Murray PE, Garcia-Godoy F, Hargreaves KM. Regenerative endodontics: a review of current status and a call for action. J Endod 2007;33:377390.

6. Banchs F, Trope M. Revascularization of immature permanent teeth with apical periodontitis: new treatment protocol? J Endod 2004;30:196-200.

7. Ding RY, Cheung GS, Chen J, Yin XZ, Wang Q0, Zhang CF. Pulp revascularization of immature teeth with apical periodontitis: A clinical study. J Endod 2009;35:745-749.

8. Bose $R$, Nummikoski $P$, Hargreaves $K$. A retrospective evaluation of radiographic outcomes in immature teeth with necrotic root canal systems treated with regenerative endodontic procedures. J Endod 2009;35:1343-1349.

9. Jeeruphan T, Jantarat J, Yanpiset K, Suwannapan L, Khewsawai P, Hargreaves KM. Mahidol study 1: comparison of radiographic and survival outcomes of immature teeth treated with either regenerative endodontic or apexification methods: a retrospective study. J Endod 2012;38:1330-1336.

10. Nosrat A, Seifi A, Asgary S. Regenerative endodontic treatment (revascularization) for necrotic immature permanent molars: A review and report of two cases with a new biomaterial. J Endod 2011;37:562567.

11. Chen X, Bao ZF, Liu Y, Liu M, Jin XQ, Xu XB. Regenerative endodontic treatment of an immature permanent tooth at an early stage of root development: A case report. J Endod 2013;39:719-722.

12. Trevino EG, Patwardhan AN, Henry MA, Perry HG, Dybdal-Hargreaves $N$, Hargreaves KM et al. Effect of irrigants on the survival of human stem cells of the apical papilla in a platelet-rich plasma scaffold in human root tips. J Endod 2011; 37:1109-1115.

13. Huang GT, Sonoyama W, Liu Y, Liu H, Wang S, Shi S. The hidden treasure in apical papilla: the potential role in pulp/dentin regeneration and bioroot engineering. J Endod 2008;34:645-651.
14. Sonoyama W, Liu Y, Yamaza T, Tuan RS, Wang S, Shi S et al. Characterization of the apical papilla and its residing stem cells from human immature permanent teeth: a pilot study. J Endod 2008;34:166171.

15. Seo BM, Miura M, Gronthos S, Bartold PM, Batouli S, Brahim J et al. Investigation of multipotent postnatal stem cells from human periodontal ligament. Lancet 2004;364:149-155.

16. Lei L, Chen Y, Zhou R, Huang X, CaiZ. Histologic and immunohistochemical findings of a human immature permanent tooth with apical periodontitis after regenerative endodontic treatment. J Endod 2015;41:1172-1179.

17. Haynesworth SE, Goshima J, Goldberg VM, Caplan Al. Characterization of cells with osteogenic potential from human marrow. Bone 1992;13:81-88.

18. Lovelace TW, Henry MA, Hargreaves KM, Diogenes A. Evaluation of the delivery of mesenchymal stem cells into the root canal space of necrotic immature teeth after clinical regenerative endodontic procedure. J Endod 2011;37:133-138

19. Martin DE, De Almeida JF, Henry MA, Khaing ZZ, Schmidt CE, Teixeira FB et al. Concentration-dependent effect of sodium hypochlorite on stem cells of apical papilla survival and differentiation. J Endod 2014;40:5155.

20. Bezgin T, Sönmez H. Review of current concepts of revascularization/ revitalization. Dent Traumatol 2015;31:267-273.

21. Tanomaru-Filho M, Lima RKP, Nakazone PA, Tanomaru MG. Use of computerized tomography for diagnosis and follow-up after endodontic surgery: clinical case report with 8 years of follow-up. Oral Surg Oral Med Oral Pathol Oral Radiol Endod 2010;109:629-633

22. Chen MY, Chen KL, Chen CA, Tayebaty F, Rosenberg PA, Lin LM. Responses of immature permanent teeth with infected necrotic pulp tissue and apical periodontitis/abscess to revascularization procedures. Int Endod J 2012:45:294-305.

Received April 15, 2015

Accepted October 5, 2015 